\title{
Portal vein wedge resection and patch venoplasty using autologous and homologous vein grafts during surgery for hepatobiliary malignancies
}

\author{
Byeong-Gon Na, Shin Hwang, Dong-Hwan Jung, Sung-Gyu Lee
}

Department of Surgery, Asan Medical Center, University of Ulsan College of Medicine, Seoul, Korea

\begin{abstract}
Obtaining tumor-free resection margins is one of the most important factors for achieving favorable prognosis of patients undergoing resection for hepatobiliary malignancies. In this study, we present our experience of portal vein (PV) wedge resection and patch venoplasty using autologous or homologous vessel grafts for resecting perihilar cholangiocarcinoma, hepatocellular carcinoma, and distal bile duct cancer. Case 1 was 68-year-old male patient with type IV perihilar cholangiocarcinoma who underwent central bisectionectomy with caudate lobectomy and bile duct resection, and PV wedge resection and patch venoplasty with a cryopreserved iliac vein allograft patch. This patient survived 14 months after surgery. Case 2 was 77 -year-old male patient with type IIIA perihilar cholangiocarcinoma who underwent left medial sectionectomy with caudate lobectomy, bile duct resection, and PV wedge resection and patch venoplasty with a cryopreserved iliac vein allograft patch. This patient survived 17 months after surgery. Case 3 was 54 -year-old male patient with hepatitis B virus-associated liver cirrhosis and hepatocellular carcinoma with PV tumor thrombus who underwent left hepatectomy. The PV wall defect was repaired with an autologous greater saphenous vein patch. This patient survived 11 months after surgery. Case 4 was 65 -year-old female patient with distal bile duct cancer who underwent pylorus-preserving pancreaticoduodenectomy, and main PV wedge resection and patch venoplasty with a cryopreserved iliac artery allograft patch. This patient survived 21 months after surgery. In conclusion, PV wedge resection and patch venoplasty can be used to facilitate complete tumor resection in patients undergoing various extents of surgical resection for hepatobiliary malignancies.
\end{abstract}

Key Words: Portal vein; Allograft; Autografts; Greater saphenous vein; Vascular reconstruction

\section{INTRODUCTION}

Hepatobiliary malignancies can directly invade the portal vein $(\mathrm{PV})$ due to their physical proximity to the PV. Obtaining tumor-free resection margins is one of the most important factors for achieving favorable prognosis of patients undergoing resection for hepatobiliary malignancies [1-3]. PV resection

Received: January 3, 2021, Revised: January 6, 2021,

Accepted: January 7, 2021

Corresponding author: Shin Hwang

Department of Surgery, Asan Medical Center, University of Ulsan College of Medicine, 88 Olympic-ro 43-gil, Songpa-gu, Seoul 05505, Korea

Tel: +82-2-3010-3930, Fax: +82-2-3010-6701, E-mail: shwang@amc.seoul.kr ORCID: https://orcid.org/0000-0002-9045-2531

Copyright (C) The Korean Association of Hepato-Biliary-Pancreatic Surgery This is an Open Access article distributed under the terms of the Creative Commons Attribution Non-Commercial License (http://creativecommons.org/licenses/by-nc/4.0) which on-commercial use, distribution, and reproduction in any medium, provided the original permits unrestricted work is properly cited. combined with hepatectomy has been frequently performed for patients with perihilar cholangiocarcinoma. Common PV resection-reconstruction methods include PV wedge resection combined with primary repair, and segmental PV resection with end-to-end anastomosis or interposition vessel graft [4]. Patch venoplasty following PV wedge resection has been rarely performed due to of its indications. We have previously presented our experience of hilar PV wedge resection and allograft patch venoplasty in patients undergoing bile duct resection [5]. We herein present our experience of PV wedge resection and patch venoplasty using autologous or homologous vessel grafts for surgical resection of perihilar cholangiocarcinoma, hepatocellular carcinoma, and distal bile duct cancer.

\section{CASE PRESENTATION}

\section{Case 1}

A 68-year-old male patient was referred to our institution 
with the diagnosis of Bismuth-Corlette type IV perihilar cholangiocarcinoma (Fig. 1A, 1B). The tumor was centrally located at the hepatic hilum. The size of the left lateral section was too small to safely perform right trisectionectomy even after considering right PV embolization. Thus, it was decided that the patient would undergo central bisectionectomy.

During hilar dissection, we found that the tumor invaded the confluence portion of the PV bifurcation and the right anterior PV branch (Fig. 2A). Thus, the invaded PV portion and the right anterior PV branch were excised elliptically. The wall defect at the hilar PV was repaired with a cryopreserved iliac vein allograft patch (Fig. 2B, 2C). Afterwards, segments IV, V, VIII and caudate lobe were resected through central bisectionectomy and caudate lobectomy (Fig. 2D). Roux-en-Y hepaticojejunostomy was performed to the right posterior section and left lateral section ducts.

The pathology report revealed that the tumor was a $3.5 \mathrm{~cm}$ sized poorly differentiated adenocarcinoma (Fig. 1C). All bile duct resection margins were tumor-negative. Regarding the depth of invasion, the tumor extended beyond the bile duct with involvement of the liver parenchyma and the perihilar soft tissue. Lymphovascular invasion and perineural invasion were also present. Two of five resected lymph nodes showed metastasis. According to the 8th edition of the American Joint Committee on Cancer (AJCC) [6], the extent of the tumor was pT4N1M0, which was regarded as stage IIIC.
This patient recovered uneventfully from the surgery. The PV reconstruction site appeared to be slightly stenotic on follow-up computed tomography scans (Fig. 1D). After the surgery, adjuvant chemotherapy was performed. However, multiple intrahepatic recurrence occurred at 8 months after the surgery. This patient passed away at 14 months after the surgery due to rapid progression of tumor metastasis.

\section{Case 2}

A 77-year-old male patient was referred to our institution with the diagnosis of Bismuth-Corlette type IIIA perihilar cholangiocarcinoma (Fig. 3A, 3B). The size of the left liver appeared to be too small to safely perform right hepatectomy even after considering right portal and hepatic vein embolization [7]. The general condition of this patient was relatively poor with old age. Thus, we decided to perform parenchyma-preserving hepatectomy.

During hilar dissection, we found that the tumor invaded the transverse portion of the left PV. Thus, the PV-invading tumor was meticulously detached from the left PV. Afterwards, the segment IV and the caudate lobe were resected through left medial sectionectomy and caudate lobectomy. After obtaining a wide-enough operative field through removal of the segment IV and the caudate lobe, the tumor-invaded left PV wall was elliptically excised. The defect in the PV wall was repaired with a cryopreserved iliac vein allograft patch (Fig. 4). Roux-en-Y
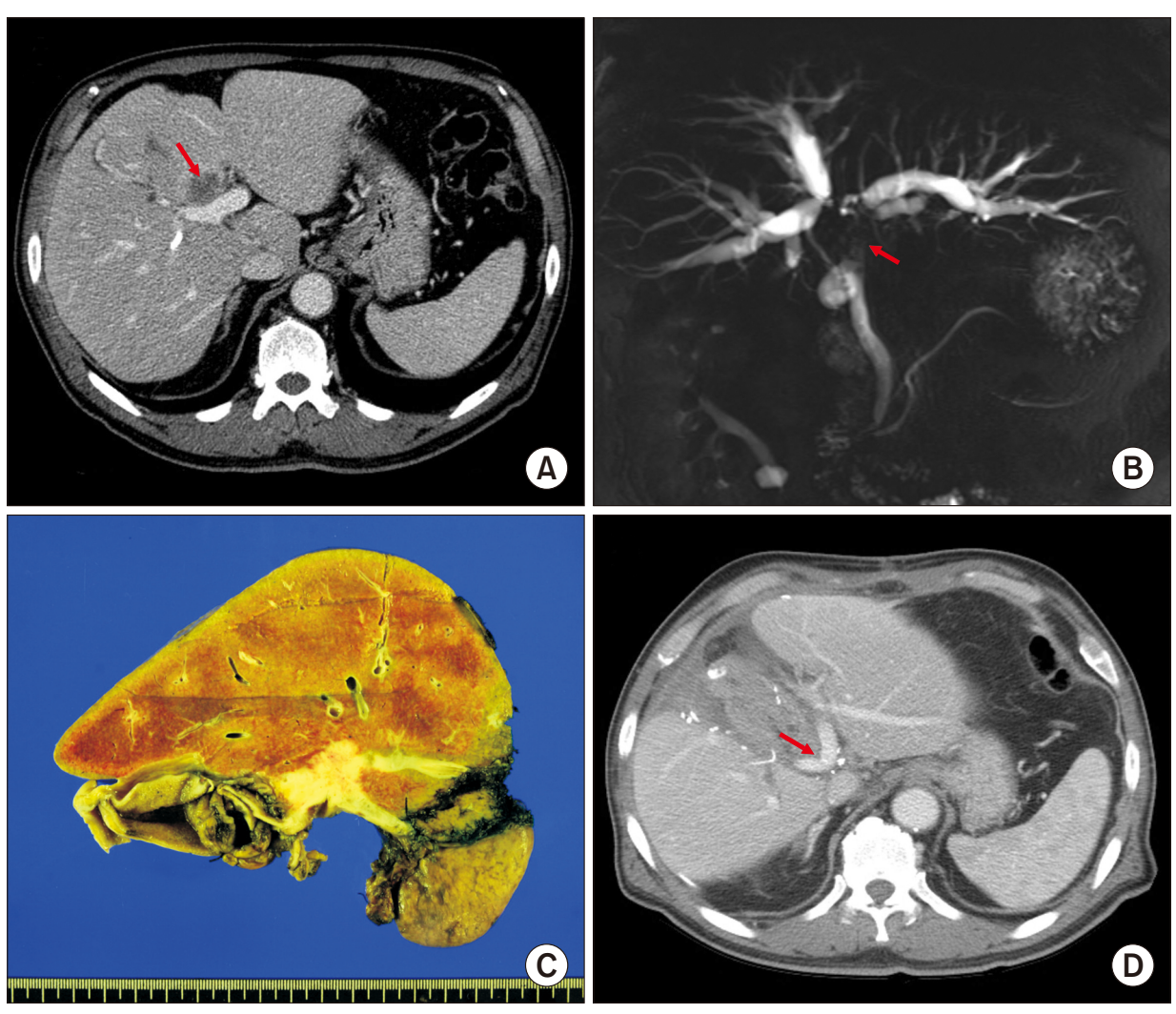

Fig. 1. Perioperative findings of Case 1. (A) Preoperative computed tomography scan shows advanced perihilar cholangiocarcinoma (arrow). (B) Magnetic resonance cholangiopancreatography shows extensive involvement of the hilar bile duct (arrow), indicating Bismuth-Corlette type IV tumor. (C) Gross photograph of the surgical specimen is visible after central bisectionectomy, caudate lobectomy and bile duct resection. (D) Computed tomography scan taken at 2 weeks after surgery shows slightly stenotic portal vein reconstruction site (arrow) at the portal vein confluence portion. 

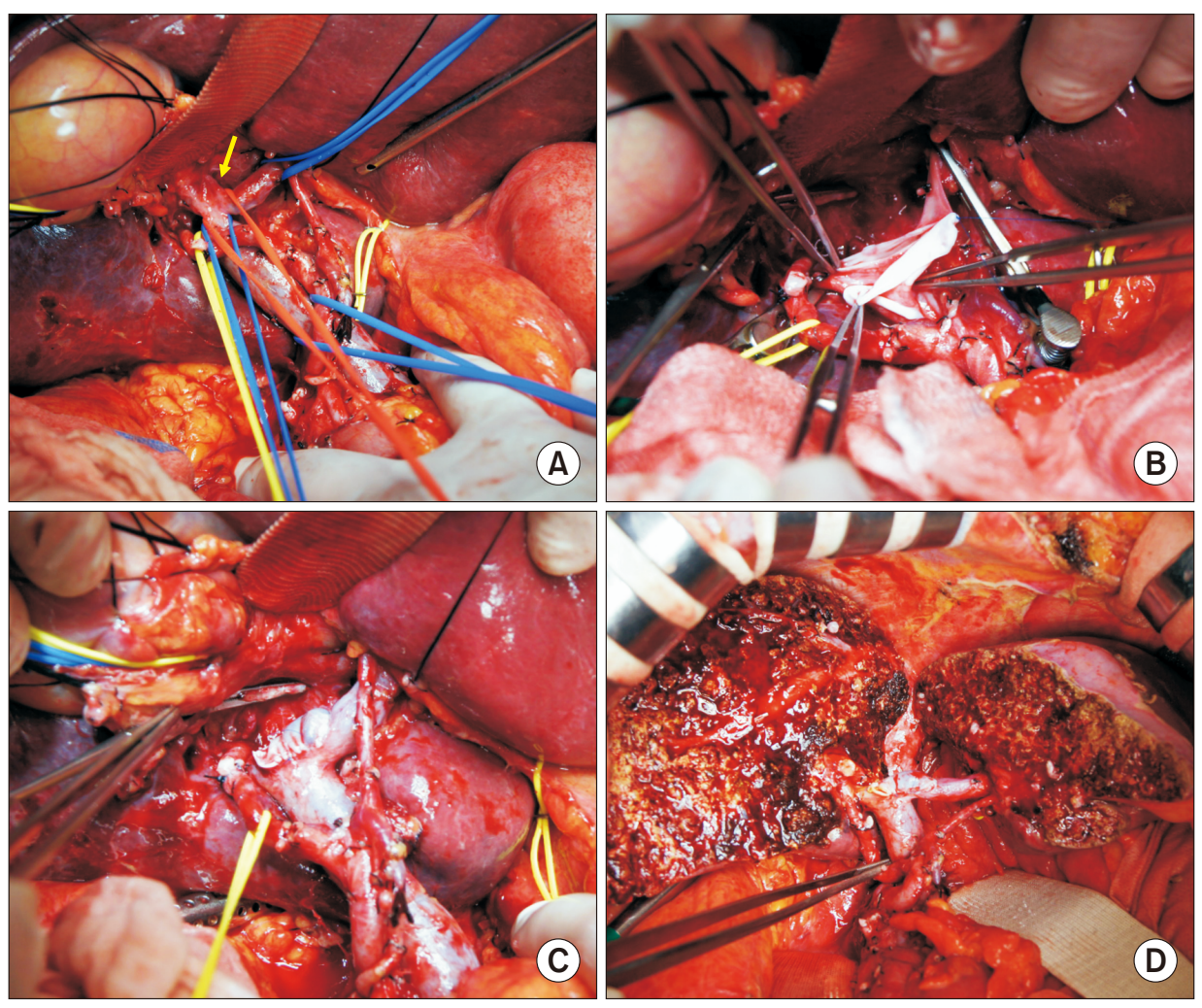

Fig. 2. Intraoperative photographs in Case 1. (A) Tumor invades the confluence portion of the portal vein (PV) bifurcation and the right anterior PV branch (arrow). (B) The invaded PV confluence portion and the right anterior PV branch are elliptically excised and the wall defect is repaired with a cryopreserved iliac vein allograft patch. (C) The roofing patch venoplasty is fully expanded. (D) Central bisectionectomy with caudate lobectomy and bile duct resection are completed.
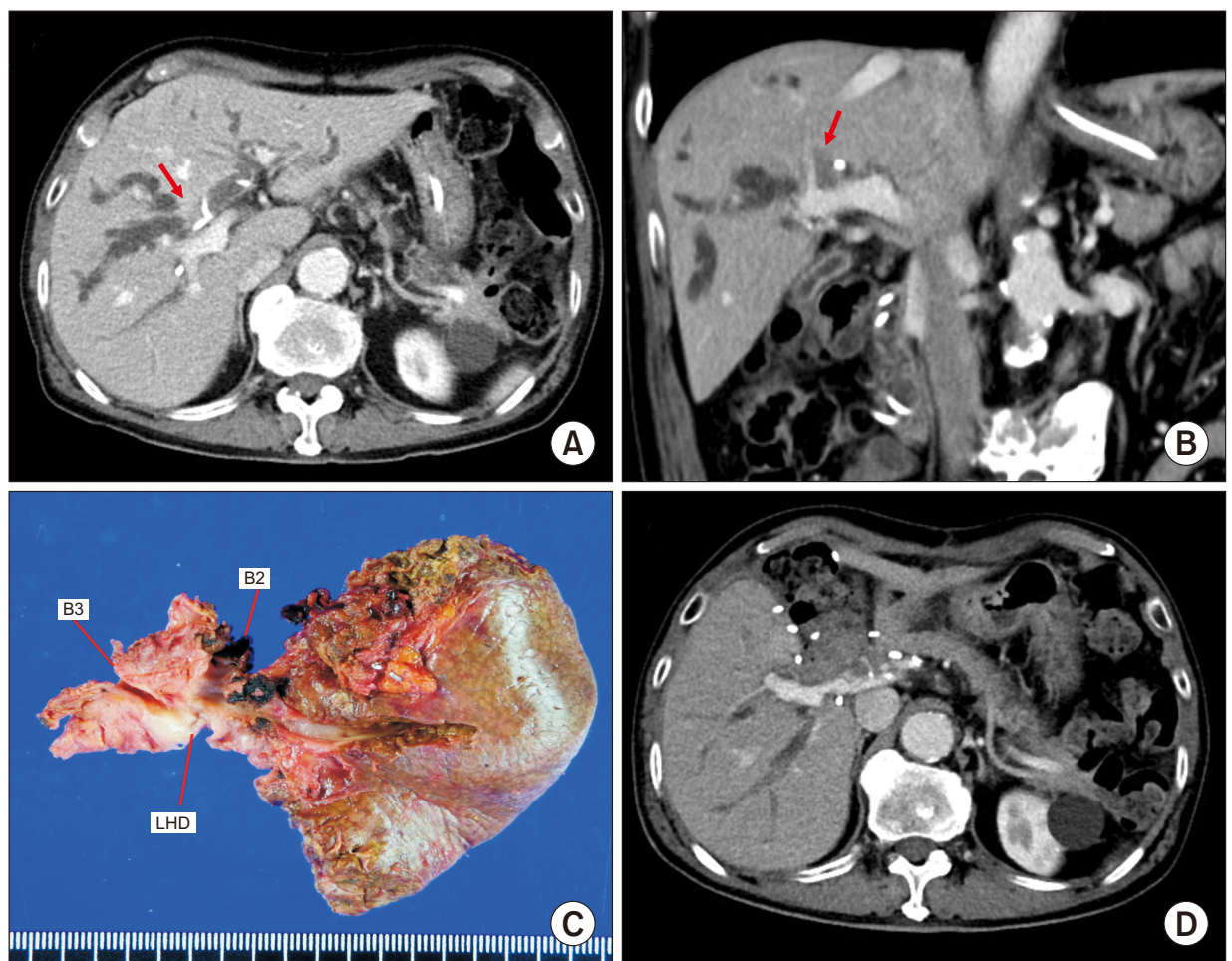

Fig. 3. Perioperative findings of Case 2. (A, B) Preoperative computed tomography scan shows advanced perihilar cholangiocarcinoma (arrows). (C) Gross photograph of the surgical specimen is visible after left medial sectionectomy, caudate lobectomy and bile duct resection. (D) Computed tomography scan taken at 2 weeks after surgery shows no abnormal findings. B2 and B3 indicate segment II and III ducts, respectively; LHD, left hepatic duct. 
hepaticojejunostomy was performed with the right liver and left lateral section ducts.

The pathology report revealed that the tumor was a $5 \mathrm{~cm}$ sized poorly differentiated adenocarcinoma with sarcomatoid

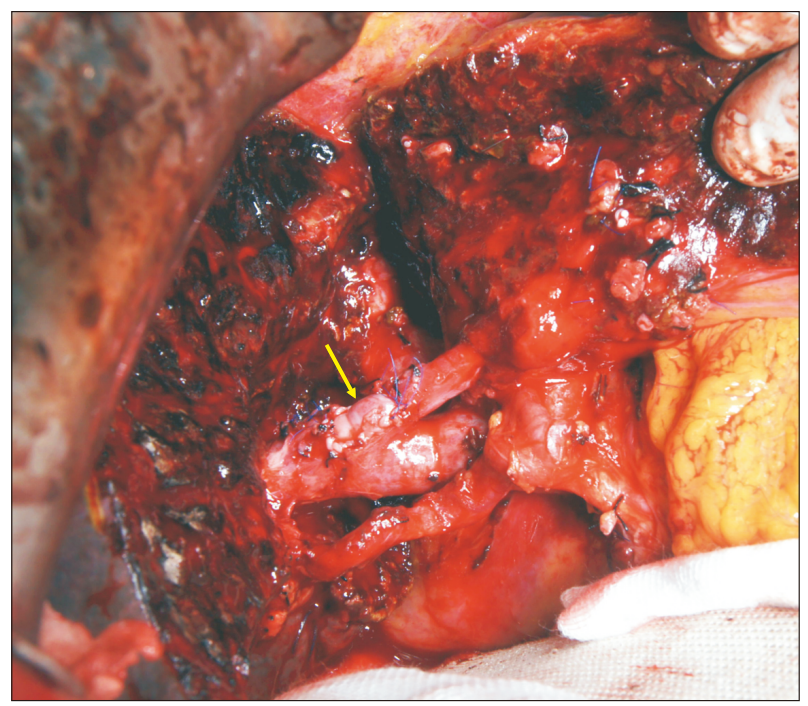

Fig. 4. Intraoperative photographs in Case 2. The tumor-invaded transverse portion of the left portal vein is elliptically excised and the wall defect is repaired with a cryopreserved iliac vein allograft patch (arrow). features (Fig. 3C). All bile duct resection margins except the periductal radial resection margin were tumor-negative. Regarding the depth of invasion, the tumor extended beyond the bile duct with involvement of the liver parenchyma and the perihilar soft tissue. Lymphovascular invasion and perineural invasion were also present. There was no tumor metastasis in any of 17 resected lymph nodes. According to the 8th AJCC [6], the extent of the tumor was pT4N0M0, which was regarded as stage IIIB.

This patient recovered uneventfully from the surgery (Fig. 3D). This patient did not undergo adjuvant therapy because of his very old age. Multiple intrahepatic recurrence occurred at 10 months after the surgery. The patient passed away at 17 months after the surgery.

\section{Case 3}

A 54-year-old male patient was referred to our institution with the diagnosis of hepatitis B virus-associated liver cirrhosis and hepatocellular carcinoma with PV tumor thrombus (Fig. $5 \mathrm{~A}, 5 \mathrm{~B})$. This patient had undergone transarterial chemoembolization for hepatocellular carcinoma at the left liver and external beam radiation therapy for PV tumor thrombus. The tumor appeared to be resectable, thus we decided to perform left hepatectomy and PV tumor thrombectomy.

During hilar dissection, we found that the PV tumor thrombus encroached the PV confluence portion, which would result in resection of the tumor thrombus-containing the left
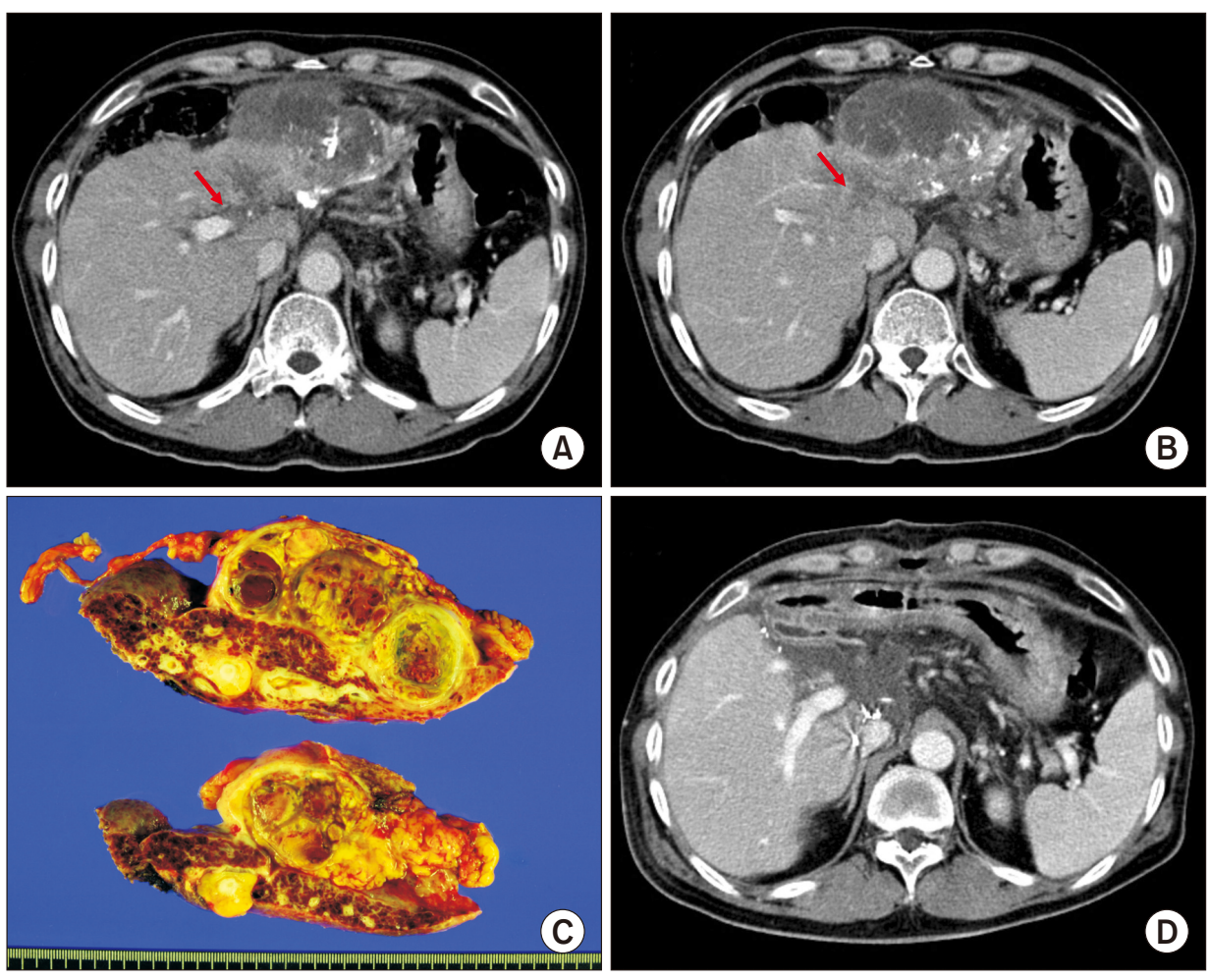

Fig. 5. Perioperative findings of Case 3. (A, B) Preoperative computed tomography scan shows hepatocellular carcinoma and portal vein tumor thrombus (arrows) at the left liver. (C) Gross photograph of the surgical specimen is visible after left hepatectomy. (D) Computed tomography scan taken at 1 week after surgery shows no abnormal findings. 
PV and leave a sizable defect (Fig. 6A, 6B). The PV wall defect would not permit primary repair. Thus, we harvested the patient's greater saphenous vein from the left thigh to create a vein patch. A 6-cm-long greater saphenous vein segment was harvested and paneled to make a rectangular patch of $3 \mathrm{~cm} \times 2$ $\mathrm{cm}$ in size (Fig. 7). After left PV resection, this autologous vein patch was then attached to the PV wall defect (Fig. 6C, 6D). Thereafter, the left liver was resected.

The pathology report revealed that the tumor was a $9 \mathrm{~cm}$ sized hepatocellular carcinoma with 99\% necrosis (Fig. 6C).
Microvascular invasion and satellite nodule were not observed. According to the 8th AJCC [6], the extent of the tumor was pT4 N0M0, which was regarded as stage IIIB.

This patient recovered uneventfully from the surgery (Fig. 6D). Multiple intrahepatic recurrence occurred at 3 months after the surgery. Three sessions of transarterial chemoembolization were performed. This patient passed away at 11 months after the surgery due to liver failure and hepatocellular carcinoma recurrence.
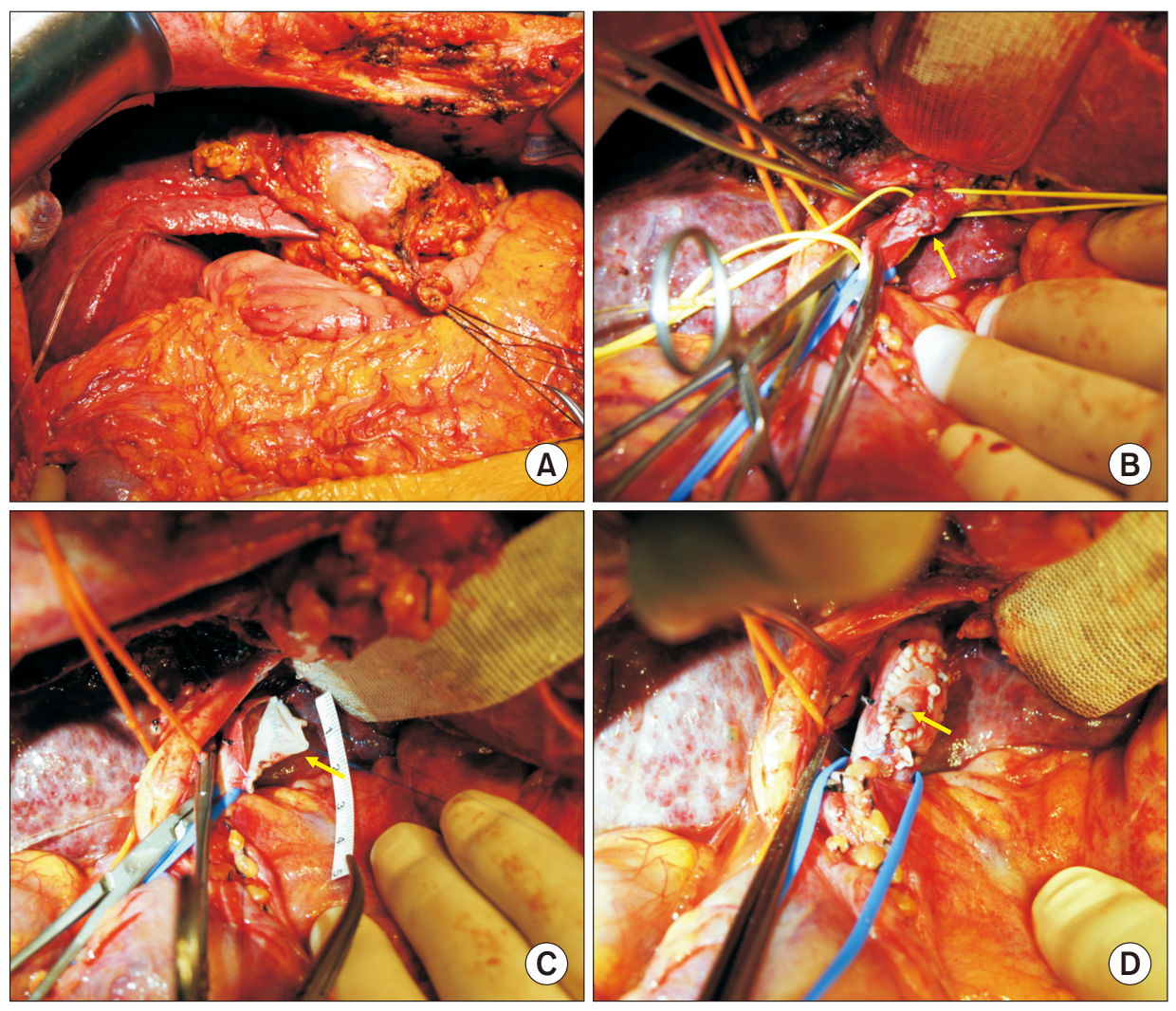

Fig. 6. Intraoperative photographs in Case 3. (A) Hepatocellular carcinoma is located at the left liver. (B) The left portal vein is transected at the confluence portion and intraluminal tumor thrombus is visible (arrow). (C, D) The portal vein wall defect is repaired with an autologous greater saphenous vein patch (arrows).
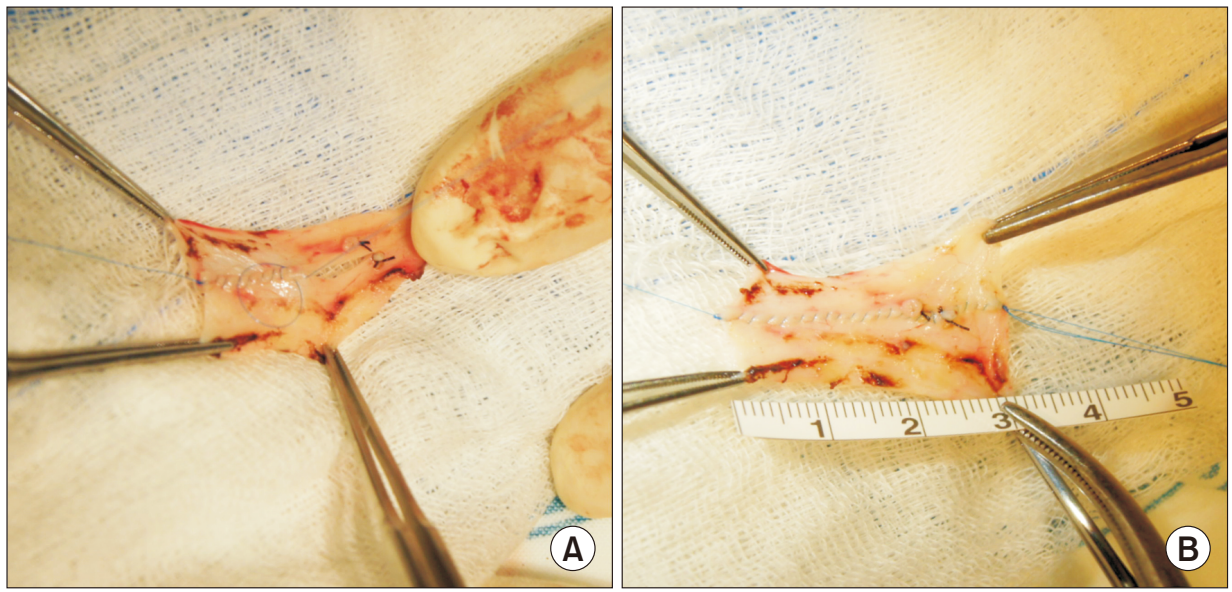

Fig. 7. Intraoperative photographs showing the greater saphenous vein patch in Case 3. Two greater saphenous vein segments are sutured (A) to make a $3 \mathrm{~cm} \times 2 \mathrm{~cm}$-sized rectangular patch $(B)$. 

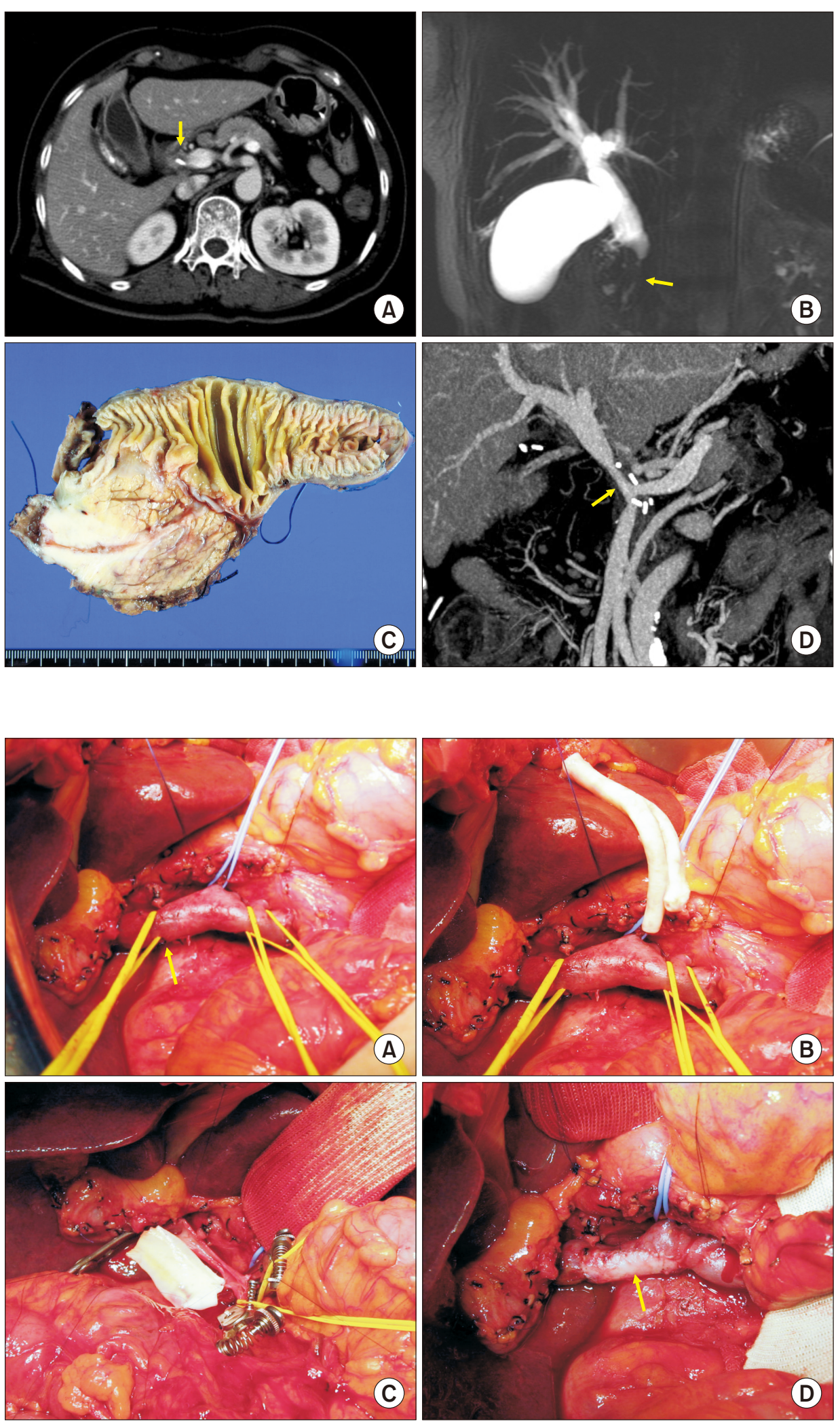

Fig. 8. Perioperative findings of Case 4 . (A) Preoperative computed tomography scan shows distal bile duct cancer (arrow). (B) Magnetic resonance cholangiopancreatography shows complete obstruction of the distal bile duct (arrow). (C) Gross photograph of the surgical specimen is visible after pylorus-preserving pancreaticoduodenectomy. (D) Computed tomography scan taken at 3 weeks after surgery shows a long stenosis at the site of portal vein reconstruction proximal to the superior mesenteric vein-splenic vein confluence portion (arrow).
Fig. 9. Intraoperative photographs in Case 4. $(A, B)$ The main portal vein is invaded by the tumor (arrow). A cryopreserved iliac artery allograft is visible. (C, D) The tumorinvaded main portal vein wall is elliptically excised and the wall defect is repaired with a cryopreserved iliac artery allograft patch (arrow). 


\section{Case 4}

A 65-year-old female patient was referred to our institution with the diagnosis of distal bile duct cancer (Fig. 8A, 8B). There was no distant metastasis. Thus, we decided to perform pylorus-preserving pancreaticoduodenectomy.

During surgery, we found that the main PV was invaded by the tumor (Fig. 9A, 9B). Because the extent of PV invasion did not permit en bloc resection, we removed the pancreatic head and the duodenum after meticulously separating the PV invasion. Thereafter, the tumor-invaded PV wall was carefully excised. The defect in the PV wall was repaired with a cryopreserved iliac artery allograft patch (Fig. 9C, 9D) because cryopreserved iliac vein allograft was not available at the tissue bank of our institution. Afterwards, the standard reconstruction technique of pylorus-preserving pancreaticoduodenectomy was performed.

The pathology report revealed that the tumor was a $5 \mathrm{~cm}$ sized adenosquamous carcinoma (Fig. 8C). All resection margins were tumor-negative. Regarding the depth of invasion, the tumor extended to the periductal soft tissue and the pancreas $(14 \mathrm{~mm})$. Perineural invasion was also present. There was no tumor metastasis in any of 10 resected lymph nodes. According to the 8th AJCC [6], the extent of the tumor was pT3N0M0, which was regarded as stage IIB.

This patient recovered uneventfully from the surgery. The $\mathrm{PV}$ reconstruction site appeared to be stenotic on follow-up computed tomography scans (Fig. 8D). This patient did not undergo adjuvant therapy because of patient refusal. Multiple lymphadenopathies occurred at 16 months after the surgery. This patient passed away at 21 months after the surgery due to rapid tumor progression.

\section{DISCUSSION}

Hepatectomy combined with PV resection for perihilar cholangiocarcinoma has been often performed to ensure surgical curability. Two high-volume meta-analyses for perihilar cholangiocarcinoma have revealed that combined PV resection is safe and feasible for treating perihilar cholangiocarcinoma $[1,2]$. As a result, $\mathrm{PV}$ resection is recommended if it can lead to obtaining tumor-free resection margins.

When performing major hepatectomy for hepatobiliary malignancies, combined PV resection can be performed using various techniques such as wedge or segmental resection combined with primary repair, patch venoplasty, and vessel interposition. The extent of PV resection and reconstruction techniques vary depending on the site and extent of PV invasion. Meanwhile, when central hepatectomy is performed, segmental resection of the main or hemihepatic PV branch is particularly technically demanding due to the difficulty in handling PV for vascular anastomosis. Consequently, the preferred procedure is wedge resection of the involved PV portion and subsequent patch venoplasty, as presented in Cases 1 and 2 of this study.
Unlike primary repair or end-to-end anastomosis, roofing patch venoplasty essentially requires a vessel patch. A coldstored fresh vein allograft such as an external iliac vein is the best material for patch venoplasty. However, such allograft veins are usually not available in most centers worldwide. The next suitable patch material is a cryopreserved vein allograft, which is stored at the tissue bank. In Cases 1 and 2, we used cryopreserved external iliac vein allograft patches, which were preserved at the tissue bank of our institution. On the contrary, in Case 4, a cryopreserved external iliac artery allograft had to be used because an iliac vein allograft was not available at that time.

In the situation where vein allografts are unavailable for use during surgery, the use of an autologous vein patch can be considered. As shown in Case 3, the patient's own greater saphenous vein segment could be converted to a sizable paneled vein patch through a longitudinal incision of the vein and suturing to double the patch width $[8,9]$. Under most circumstances, we do not recommend the use of any prosthetic vascular graft for $\mathrm{PV}$ patch venoplasty because it is much more thrombogenic than autologous or homologous vein grafts. The PV is a medium-velocity high-flow vessel. Thus, anticoagulation is usually not needed if the luminal diameter of the PV is maintained. On the contrary, the development of focal stenosis can lead to a high risk of PV thrombosis. Thus, a hemodynamics-compliant design is essential for PV resection and reconstruction.

Regarding roofing patch venoplasty as shown in Cases 1 and 2, it is important to make the PV wall defect as small as possible. The size of the vein patch should be either twice or three times larger than the defect size at the PV wall. The patch should be large enough to make the patch roof redundant.

The availability of vein allografts facilitates combined vascular resection and reconstruction during aggressive surgery for hepatobiliary malignancies and living donor liver transplantation $[4,10,11]$. Currently, cryopreserved femoral vein and artery allografts are commercially available through the Korea Public Tissue Bank. All human tissues were donated and stored at the tissue bank after informed consent was obtained from donors' family members. All procedures for vascular tissue procurement and processing were complied with Korean legislation and conformed to the ethical and safety concerns for therapeutic use [12].

Complete resection of the PV invasion through PV wedge resection and patch venoplasty was successfully performed in all 4 cases in the present study. However, all 4 patients passed away within 2 years due to tumor recurrence and rapid tumor progression. Perihilar cholangiocarcinoma with PV invasion is regarded as T4 tumor stage based on the 8th AJCC staging system [6]. Macrovascular PV thrombosis is one of the most important risk factors for hepatocellular carcinoma [13]. For distal bile duct cancer, PV invasion is also a significant risk factor, although the prognostic impact of PV invasion is not listed in the 7 th and the 8 th AJCC staging systems $[6,14,15]$. 
In conclusion, we propose that PV wedge resection and patch venoplasty can be used to facilitate complete tumor resection in patients undergoing various extents of surgical resection for hepatobiliary malignancies.

\section{CONFLICT OF INTEREST}

No potential conflict of interest relevant to this article was reported.

\section{ORCID}

Byeong-Gon Na, https://orcid.org/0000-0002-3150-4645

Shin Hwang, https://orcid.org/0000-0002-9045-2531

Dong-Hwan Jung, https://orcid.org/0000-0001-5984-023X

Sung-Gyu Lee, https://orcid.org/0000-0001-9161-3491

\section{AUTHOR CONTRIBUTIONS}

Conceptualization: SH. Data curation: BGN, DHJ. Methodology: SH, SGL. Visualization: SH. Writing - original draft: BGN, SH. Writing - review \& editing: All Authors.

\section{REFERENCES}

1. Chen W, Ke K, Chen YL. Combined portal vein resection in the treatment of hilar cholangiocarcinoma: a systematic review and meta-analysis. Eur J Surg Oncol 2014;40:489-495.

2. Wu XS, Dong P, Gu J, Li ML, Wu WG, Lu JH, et al. Combined portal vein resection for hilar cholangiocarcinoma: a meta-analysis of comparative studies. J Gastrointest Surg 2013;17:1107-1115.

3. Ebata T, Nagino M, Kamiya J, Uesaka K, Nagasaka T, Nimura Y. Hepatectomy with portal vein resection for hilar cholangiocarcinoma: audit of 52 consecutive cases. Ann Surg 2003;238:720-727.

4. Hwang S, Ha TY, Jung DH, Park JI, Lee SG. Portal vein interposition using homologous iliac vein graft during extensive resection for hilar bile duct cancer. J Gastrointest Surg 2007;11:888-892.

5. Kim SM, Hwang S. Hilar portal vein wedge resection and patch venoplasty in patients undergoing bile duct resection for hepatobiliary malignancy: a report of two cases. Ann Hepatobiliary Pancreat Surg 2021;25:132-138.

6. Amin MB, Edge S, Greene F, Byrd DR, Brookland RK, Washington MK, et al. AJCC cancer staging manual. 8th ed. New York: Springer, 2017.

7. Hwang S, Ha TY, Ko GY, Kwon DI, Song GW, Jung DH, et al. Preoperative sequential portal and hepatic vein embolization in patients with hepatobiliary malignancy. World J Surg 2015;39:2990-2998.

8. Sakamoto Y, Yamamoto J, Saiura A, Koga R, Kokudo N, Kosuge $\mathrm{T}$, et al. Reconstruction of hepatic or portal veins by use of newly customized great saphenous vein grafts. Langenbecks Arch Surg 2004;389:110-113.

9. Lin CC, Hsieh CE, Chen YL. Great saphenous vein harvesting for venous outflow reconstruction in living donor liver transplantation a minimally invasive refinement of the conventional procedure. Int J Surg 2013;11:249-252.

10. Hwang S, Ko GY. Right trisectionectomy with en bloc portal vein resection for cholangiocarcinoma after preoperative stenting for main portal vein occlusion. Ann Hepatobiliary Pancreat Surg 2020;24:174181.

11. Namgoong JM, Hwang S, Oh SH, Kim KM, Park GC, Ahn CS, et al. Living-donor liver transplantation with inferior vena cava replacement in an infant recipient with advanced hepatoblastoma. Ann Hepatobiliary Pancreat Surg 2020;24:72-77.

12. Kwon H, Kwon H, Hong JP, Han Y, Park H, Song GW, et al. Use of cryopreserved cadaveric arterial allograft as a vascular conduit for peripheral arterial graft infection. Ann Surg Treat Res 2015;89:51-54.

13. Hwang S, Moon DB, Kim KH, Ahn CS, Song GW, Jung DH, et al. Prognostic accuracy of the ADV score following resection of hepatocellular carcinoma with portal vein tumor thrombosis. J Gastrointest Surg 2020. https://doi.org/10.1007/s11605-020-04800-6 [in press]

14. Edge SB, Byrd DR, Compton CC, Fritz AG, Greene FL, Trotti A III. AJCC cancer staging manual. 7th ed. New York: Springer, 2010.

15. Maeta T, Ebata T, Hayashi E, Kawahara T, Mizuno S, Matsumoto N, et al. Pancreatoduodenectomy with portal vein resection for distal cholangiocarcinoma. Br J Surg 2017;104:1549-1557. 